\title{
Hyaluronic acid application vs arthroscopy in treatment of internal temporomandibular joint disorders
}

\author{
Stasko J, Statelova D, Janickova M, Mikuskova K, Bacinsky M, Sokol J, Frlickova Z, \\ Hvizdos D, Malachovsky I
}

Department of Oral and Maxillofacial Surgery, Comenius University in Bratislava, Jessenius Faculty of Medicine in Martin, University Hospital Martin, Slovakia. malachovsky@unm.sk

\begin{abstract}
AIMS: The goal of this study was to compare the treatment outcome after two different treatment modalities in cases of disc displacement of the temporomandibular joint diagnosed via magnetic resonance technique, namely surgical procedure in form of arthroscopic lysis and lavage vs. hyaluronic acid superior joint space application.

MATERIALS AND METHODS: Magnetic resonance imaging was performed before and one year after treatment. Simultaneously, pre- and post-treatment visual analogue scales and maximal mouth opening distance were documented.

RESULTS: In our study, out of the total of 145 patients who were initially diagnosed with magnetic resonance imaging to have internal derangements and treated with arthroscopic lysis and lavage or hyaluronic acid application, we observed progression of internal derangement in 27 cases (18.6\%), improvement in 37 cases $(25.5 \%)$ and stationary state in 81 patients (55.9\%). Both treatment methods significantly improved the followed maximal mouth opening distance and visual analogue scale, as confirmed upon the 12-month follow-up examination. Maximal mouth opening was $4.7 \mathrm{~mm}$ for hyaluronic acid application and $12.2 \mathrm{~mm}$ for arthroscopic lysis and lavage $(p<0.005)$. The mean visual analogue scale values decreased from 6.2 to 2.1 for hyaluronic acid application and from 6.9 to 1.2 for arthroscopic lysis and lavage $(p<0.005)$.

CONCLUSION: Our study shows that both arthroscopic lysis and lavage and hyaluronic acid injections are effective and safe in the treatment of disc displacement in the temporomandibular joint (Tab. 3, Ref. 30). Text in PDF www.elis.sk.

KEY WORDS: arthroscopic lysis and lavage; hyaluronic acid intraarticular application; internal derangement of temporomandibular joint; magnetic resonance imaging.
\end{abstract}

\section{Introduction}

Magnetic resonance imaging (MRI) is considered to be the golden standard in diagnosing the intraarticular pathologies. Due to its capability of high-quality imaging of soft tissues of the temporomandibular joint, (TMJ) it is a perfect facility for diagnosing the condylar disc pathologies as well as for determining the disc displacement severity. The standard MRI technique of TMJ uses oblique sagittal and oblique coronal images. The dynamic imaging is performed in straight sagittal orientation along the anticipated path of the condylar motion (7). The position of the posterior band of the disc in sagittal mouth-closed images is known as a standard

Department of Oral and Maxillofacial Surgery, Comenius University in Bratislava, Jessenius Faculty of Medicine in Martin, University Hospital Martin, Slovakia

Address for correspondence: I. Malachovsky, Department of Oral and Maxillofacial Surgery, Comenius University in Bratislava, Jessenius Faculty of Medicine in Martin, University Hospital Martin, Kollárova 2, SK-036 01 Martin, Slovakia.

Phone: +0434203357

Acknowledgement: The study was supported by Grant of the Ministry of Health of the Slovak Republic 2018/14-UKMT-10. reference point for normal superior disc position. It is normally located at the 11 o'clock or more posterior clockwise position of the superior condyle surface.

If the disc is malpositioned in relation to the mandibular condyle and articular eminence (disc displacement), this state is known as internal derangement (ID) of TMJ. The most frequent displacement type is anterior disc displacement (DD), which can be seen in oblique sagittal images. In most large MRI studies, about $80 \%$ of TMD patients referred for diagnostic MRI imaging of the TMJ have resulted with the diagnosis of disc displacement (13). Mouth-opened sagittal images give us information about disc mobility. According to disc reduction ability, reducing (DDR) or non-reducing disc dislocations (DDwR) can be distinguished and the severity of the defect determined. The diagnostic accuracy of MRI on fresh autopsy material using oblique sagittal and oblique coronal sections has been found to be $95 \%$ and $93 \%$ in determining the disc position and bone status, respectively (13).

Arthroscopy (AS) of TMJ is a diagnostic-therapeutic miniinvasive surgery technique first used by Onishi in 1974. It allows direct visual inspection of the internal TMJ structures through arthroscope. When extended, the therapeutic abilities of AS are used (i.e. shaver, laser, suturing, etc). This procedure is usually 
referred to as operative arthroscopy. Arthroscopic lysis and lavage (ALL) are modified AS protocol procedures described in 1986 by Sanders for the treatment of chronic closed lock of TMJ. The mechanism of action of this procedure is usually proposed as breaking down the adhesions by sweeping-through catheter maneuvers reducing the surface tension and friction. At the same time the lavage washes out the intra-articular inflammatory substances and degradation products, increases mobility, disrupts adhesions, normalizes intraarticular pressure, improves disc mobility and releases the disc $(9,16,19,21,29)$.

Hyaluronic acid (HA) is a biological molecule found naturally in the TMJ and produced by synovial cells. It provides viscoelastic properties to synovial fluid. HA was first used in form of intraarticular application (IA) in the treatment of osteoarthrosis of the knee in 1971. HA can stimulate natural HA production, inhibit osteoarthritis progression, and reduce fibrous tissue proliferation (17). The proposed mechanism of action is down-regulation of matrix metalloproteinase- 3 and IL- $1 \beta$ in synovium during early development of osteoarthritis (10). IA HA is known for shock absorption, joint lubrication, anti-inflammatory effects, chondroprotection, proteoglycan synthesis and cartilage matrix alterations (3). An injection of HA into the superior joint compartment could modify the synovial fluid constitution and improve the microcondition within the joint space. It can relieve the clinical symptoms in the short term, and possibly prevent TMJ disease progression in the long term (14).

\section{Material and methods}

This retrospective study was approved by the Ethics Committee of Jessenius Faculty of Medicine, Comenius University (JFM CU) (EK 49/2019), and as a real retrospective local clinical trial it obtained an exemption from the demands of signed informed consent.

Inclusion criteria encompass patients who underwent TMJ arthroscopy or HA joint injection of upper joint space due to positive ID clinical symptoms confirmed with initial MRI. Most of the patients were previously treated in a non-invasive way with unsatisfactory results. Patients were of any gender and ethnic group, and aged above 18 years. The monitoring period was set between January 1st, 2014 and January 1st, 2019. The contralateral mostly asymptomatic TMJs were used as the control group.

The exclusion criteria were as follows: myofascial pain dysfunction syndrome, systemic or autoimmune disease, extensive degenerative joint disease, polyarthritis, known birth defects of TMJ, past local corticoid application, major joint trauma, previous joint surgery, contralateral joint ID more severe than 2nd degree according to Wilkes, missing clinical or MRI control in designed intervals, poor compliance or non-compliance, and age under 18 years.

In total, 145 patients met the inclusive criteria. Two study groups were assembled. The first group comprised 46 patients treated with ALL. The second group comprised 99 patients treated with HA joint space injection.

All patients underwent preprocedural clinical examination. This was based on Diagnostic Criteria for Temporomandibu- lar Disorders (DC/TMD) axis I revised by Schiffman in 2014, the main clinical diagnostic guide for establishing the diagnosis and treatment protocol for TMDs, including discopathy (28). This encompassed the collection of visual analogue scale (VAS) score, TMJ sounds auscultation, TMJ and masticatory muscles palpation, maximal mouth opening (MMO) and basic radiological assessment through orthopantomogram. Nevertheless, in our study we used only 2 indicators as variables, of which one is objective (MMO) and one is subjective (VAS).

To evaluate the pain level, the patients were asked to rate their pain on VAS from 0 to 10 , ( 0 for the absence of pain and 10 for the maximum pain). MMO was measured as a distance (in $\mathrm{mm}$ ) between the incisal edges of the upper and lower patient's central incisors in the maximal abduction position of mandible.

HA $/ 1 \mathrm{ml} 0.8 \%$ solution, "Sinovial Mini", IBSA Farmaceutici Italia Srl/ was administered in a series of a total of three IA injections in one-week intervals. There was no special recommendation given to the patient after the procedure. ALL was realized during a three-day hospitalization period at the Martin University Hospital Department of Stomatology and Maxillofacial Surgery. The surgery was performed on the second day after admission under general anesthesia with naso-tracheal intubation and mouth held in a wide-open position. The procedure was done by a skilled senior surgeon using an endoscope, $1.9 \mathrm{~mm}$ in diameter. After surgery, the patients were instructed about strict soft diet for 7 days, and analgesic options, as well as relaxing, atraumatic and antiseptic local and systemic measures. In most cases, the clinical follow up examinations were carried out one week and two months after surgery. The clinical examination study data were collected six and twelve months after the procedural examinations for both groups.

MRI of TMJ was conducted in each patient prior to the procedure and one year after it. MRI was performed using 0.25 Tesla MRI scanner (ESAOTE S - scan) with dedicated double-sided TMJ coil, 3-mm sections thickness, $150 \times 150 \mathrm{~mm}$ field of view, $256 \times 256$ matrix, $0.859 \mathrm{~mm}$ pixel dimension, and FSE PD technique /fast spin echo proton density/ TR 2030 / TE 25.0 ms. The MRI protocol was based on oblique sagittal and oblique coronal sections in closed and open mouth positions. All MRI images were assessed by an independent senior radiologist according to generally established assessment criteria. The initial and one-year follow-up MRI results were compared and the changes in state were classified as improved, stationary or progressive.

The statistical analysis was performed with the SPSS software (ver. 22.0 for MAC OSX; SPSS, Chicago, IL, USA). The data on age, VAS scores, MRI findings and MMO values were reported as medians. The sex distribution is reported as the ratio of males to females. These parameters were statistically compared between the two treatment groups by using Mann-Whitney test. P values less than 0.05 were considered statistically significant.

\section{Results}

At our department, we examined 2,684 patients with the diagnosis of TMD between 2014 and 2019. The mean age of patients was 44.5 years. Women accounted for $80.22 \%$ (2,153 patients) 
Tab. 1. Patient characteristics.

\begin{tabular}{lcc}
\hline Patient characteristics & $\begin{array}{c}\text { Hyaluronic acid } \\
\text { joint space injection }\end{array}$ & Arthroscopy \\
\hline Number of patients & 99 & 46 \\
\hline Sex & $19(19.2)$ & $10(21.3)$ \\
$\quad$ Male (\%) & $80(80.8)$ & $36(78.3)$ \\
Female (\%) & $40(18-81)$ & $34(18-72)$ \\
\hline Median age, range (years) & $91(91.9)$ & $44(96)$ \\
$\quad<65$ years $(\%)$ & $8(8.1)$ & $2(4)$ \\
$>65$ years $(\%)$ & & $19(41.3)$ \\
\hline Side & $48(48.5)$ & $27(58.7)$ \\
Right (\%) & $51(51.5)$ & \\
Left $(\%)$ & & \\
\hline
\end{tabular}

and men accounted for $19.78 \%$ (531 patients) with a ratio exceeding 4:1. ALL was performed in 112 patients. Our inclusion criteria for arthroscopic surgery were met in 46 cases. Superior joint space application of HA was performed in 644 patients. Our inclusion criteria for superior joint space application of HA were as gender, age, and side (right or left) of the studied joint can be seen in Table 1.

Both clinical parameters, MMO and VAS, were improved at 6- and 12-month follow-up examinations as depicted in Table 2. The increase in mean MMO distance was $4.2 \mathrm{~mm}$ for IA HA application and $10.0 \mathrm{~mm}$ for ALL at the six-month follow-up and 4.7 $\mathrm{mm}$ for IA HA and $12.2 \mathrm{~mm}$ for ALL at the twelve-month followup. The difference at the twelve-month follow up of MMO was statistically significant $(p<0.005)$. In six months, the mean VAS values decreased from 6.2 to 1.2 for IA HA application and from 6.9 to 0.9 for ALL. In twelve months, the VAS values decreased to 2.1 for IA HA application and to 1.2 for ALL. The difference at the twelve-month follow up of VAS value was statistically significant $(\mathrm{p}<0.005)$. The subgroup analysis of patients in both ALL and HA AI groups is depicted in Table 3. The comparison of preoperative/basal and twelve-month follow-up data on VAS met in 99 cases. Precise distribution of patients' characteristics

showed an improvement by $95.7 \%$ for ALL and by $77.8 \%$ for HA IA application, and the improvement in MMO by $91.3 \%$ for ALL and by $59.6 \%$ for HA IA application (response criteria according to American Association of Oral and Maxillofacial Surgeons; successful treatment VAS $£ 3 \mathrm{~mm}, \mathrm{MMO}^{3} 35 \mathrm{~mm}$ ), respectively, while the results were statistically significant $(\mathrm{p}<0.005, \mathrm{p}<0.01$, respectively) and in favor of ALL. We observed a treatment success by $77.8 \%$ in VAS and by $59.6 \%$ in MMO for HA IA application, by $95.7 \%$ in VAS and by $91.3 \%$ in MMO for ALL, respectively (according to the criteria of American Association of Oral and Maxillofacial Surgeons; successful treatment VAS $\leq 3 \mathrm{~mm}$, ii $\geq 35 \mathrm{~mm}$ ).

Most joints in both groups ( 90 of all 145 studied joints) were initially MRI-diagnosed as 3rd degree of ID of TMJ according to Wilkes. The Wilkes stages identified upon the initial MRI in both groups, namely stages I, II, III, and IV were found in $11(7.6 \%)$, $33(22.8 \%), 90(62 \%)$, and $11(7.6 \%)$ studied joints, respectively. Overall, of the total of 145 initially MRI-diagnosed IDs of TMJ, the 12-month follow-up MRI showed an improvement in ID in 37 cases $(25.5 \%)$, namely in 1 case of Wilkes I, 7 cases of Wilkes II, 25 cases of Wilkes III, and 4 cases of Wilkes IV. The state stayed stationary in 81 patients $(55.9 \%)$ namely in 7 cases of Wilkes I, 20 cases of Wilkes II, 47 cases of Wilkes III, 7 cases of Wilkes IV. The progression took place in 27 cases $(18.6 \%)$, namely in 3 cases of Wilkes I, 6 cases of Wilkes II, 18 cases of Wilkes III, and 0 cases of Wilkes IV). Precise distribution of pre-procedure and post-procedure MRI results for used treatment modality can be seen in Table 2 . We have documented no overall permanent complications after either of the types of treatment.

\section{Discussion}

The goal of this paper is to assess the post-treatment results of ID-affected joints preoperatively confirmed by MRI in patients after ALL surgery and HA joint space application. Degenerative changes, joint effusion, and bone marrow edema were not set as the purpose of this study due to limited analytic data caused by the retrospective nature of the study.

Tab. 2. Main results.

\begin{tabular}{|c|c|c|c|c|c|}
\hline Patient characteristics & \multicolumn{2}{|c|}{$\begin{array}{c}\text { Hyaluronic acid } \\
\text { joint space injection }\end{array}$} & \multicolumn{2}{|c|}{ Arthroscopy } & $\mathrm{p}$ value \\
\hline \multicolumn{6}{|l|}{ Mean VAS } \\
\hline Before & \multicolumn{2}{|c|}{6.2} & \multicolumn{2}{|c|}{6.9} & 0.01 \\
\hline After 6 months & \multicolumn{2}{|c|}{1.2} & \multicolumn{2}{|c|}{0.9} & 0.17 (n.s.) \\
\hline After 12 months & \multicolumn{2}{|c|}{2.1} & \multicolumn{2}{|c|}{1.2} & $0.002(<0.005)$ \\
\hline \multicolumn{6}{|l|}{ mean MMO (mm) } \\
\hline Before & \multicolumn{2}{|c|}{32.2} & \multicolumn{2}{|c|}{27.0} & 0.001 \\
\hline After 6 months & \multicolumn{2}{|c|}{36.0} & \multicolumn{2}{|c|}{37.0} & 0.06 (n.s.) \\
\hline After 12 months & \multicolumn{2}{|c|}{36.9} & \multicolumn{2}{|c|}{39.2} & 0.005 \\
\hline $\operatorname{MR}(\%)$ & Before & After & Before & After & \\
\hline 0 & $0(0)$ & $11(11.1)$ & $0(0)$ & $2(4.3)$ & \\
\hline 1 & $11(11.1)$ & $11(11.1)$ & $0(0)$ & $3(6.5)$ & \\
\hline 2 & $28(28.3)$ & $31(31.3)$ & $5(10.9)$ & $7(15.2)$ & \\
\hline 3 & $60(60.6)$ & $37(37.4)$ & $30(65.2)$ & $17(37)$ & \\
\hline 4 & $0(0)$ & $9(9.1)$ & $11(23.9)$ & $17(37)$ & \\
\hline Improvement in MRI findings ( $\%$ ) & \multicolumn{2}{|c|}{$23(23.2)$} & \multicolumn{2}{|c|}{$11(23.9)$} & \\
\hline Stable MRI findings (\%) & \multicolumn{2}{|c|}{$54(54.5)$} & \multicolumn{2}{|c|}{$24(52.2)$} & \\
\hline
\end{tabular}

$A L L$

According to the review of articles published by White et al., no definitive differences in range of motion, postoperative pain, or time required to rehabilitate the joint were found, regardless of whether advanced arthroscopic techniques or only ALL were used (30). Actually, in the results of his meta-analysis of literature concerning the management of ID, Al-Moraissi favored the treatment with ALL versus AS with regard to pain reduction (2). The main success rates for ALL when treating ID or chronic closed lock in the literature vary, but they generally support the effectiveness of ALL. 
Tab. 3. Subgroup analysis.

\begin{tabular}{|c|c|c|c|c|c|c|}
\hline \multirow{2}{*}{$\begin{array}{l}\text { Patient characteristics } \\
\text { Mean VAS }\end{array}$} & \multicolumn{2}{|c|}{ Hyaluronic acid joint space injection } & \multirow[t]{2}{*}{$\mathrm{p}$ value } & \multicolumn{2}{|c|}{ Arthroscopy } & \multirow[t]{2}{*}{$\mathrm{p}$ value } \\
\hline & Male & Female & & Male & Female & \\
\hline Before & 5.8 & 6.3 & 0.15 & 6.6 & 7.0 & 0.19 (n.s.) \\
\hline After 6 months & 1.7 & 1.1 & 0.051 & 0.8 & 0.9 & 0.86 (n.s.) \\
\hline After 12 months & 2.5 & 2.0 & 0.24 & 1.2 & 1.2 & 0.74 (n.s.) \\
\hline \multirow{5}{*}{$\begin{array}{l}\text { Mean MMO (mm) } \\
\text { Before } \\
\text { After } 6 \text { months } \\
\text { After } 12 \text { months }\end{array}$} & Male & Female & \multirow{4}{*}{$\begin{array}{l}0.2 \\
0.1 \\
0.2\end{array}$} & Male & Female & \\
\hline & 34.1 & 31.8 & & 28.7 & 26.8 & 0.1 (n.s.) \\
\hline & 37.5 & 35.6 & & 37.2 & 36.9 & 0.8 (n.s.) \\
\hline & 38.3 & 36.5 & & 41.0 & 38.6 & 0.1 (n.s.) \\
\hline & Male & Female & & Male & Female & \\
\hline \multirow{2}{*}{$\begin{array}{l}\text { Improvement in MRI findings }(\%) \\
\text { Stable MRI findings }(\%)\end{array}$} & $5(26.3)$ & $18(22.5)$ & & $5(50)$ & $6(16.7)$ & \\
\hline & $13(68.4)$ & $41(51.3)$ & & $4(40)$ & $20(55.5)$ & \\
\hline
\end{tabular}

MRI - Magnetic resonance imaging; MMO - maximal mouth opening; VAS - visual analogue scale; n.s - nonsignificant

Murakami reported that different authors studying AS described generally acceptable results of ALL for all Wilkes stages of ID. The procedure of ALL is currently preferred for stages III and IV with a good outcome but the success rates for ALL surgery were slightly lower in advanced stages of ID (20). In this regard, Bronstein and Merrill observed a success rate of $96 \%$ for stage II, $83 \%$ for stage III, $88 \%$ for stage IV, and $63 \%$ for stage V (5).

Most of our patients in both groups had ID of degree III before treatment. This is similar to the majority of subject groups in other ID studies. DDwR is the most studied ID degree because of its dramatic clinical symptoms. The patients frequently suffer from impaired function due to the closed lock.

Abboud et al published clinical results similar to ours. In his study, in intermediate/late stage of ID, the mean MMO increased from $27 \mathrm{~mm}$ preoperatively to $38 \mathrm{~mm}$ postoperatively, and VAS decreased from 7.2 preoperatively to 2.2 postoperatively (1). However, there is still no clear consensus in literature to date about the improvement in MMO and VAS values. We observed an improvement in the followed diagnostic criteria of MMO and VAS at sixand twelve-month follow-ups. According to our results, the efficacy of ALL was confirmed by significant improvement in both MMO and VAS values at the twelve -month follow up.

\section{Hyaluronic acid}

Gencer et al found that IA HA in TMJ produced better pain relief scores when compared to other anti-inflammatory agents, namely betamethasone and tenoxicam (8). Because of the severity of systemic and local side effects of corticosteroids, HA seems to be a favorable alternative. There have been lots of different HA application protocols mentioned in literature concerning the treatment of IDs of TMJ. Their differences vary from the number of IA applications to combinations with other treatment protocols.

However, in their systematic review of selected papers, Manfredini et al found that the superiority of HA injections was shown only against placebo saline injections. Nevertheless, the outcomes are comparable with those achieved with corticosteroid injections or oral appliances (18). The available literature seems to be inconclusive as to the effectiveness of HA injections with respect to other therapeutic modalities in treating TMJ disorders. There are studies that support the effectiveness of HA intraarticular therapy in both DDR and DDnR. Most of the authors report a decrease in VAS as well as in clicking. There is no consensus concerning the improvement in MMO. Yet, some positive results concerning VAS and MMO in the diagnosis of ID were documented in studies by Sato et al, Long et al and Korkmaz et al (11, 15, 24, 25).

Aforementioned results of other authors are in accordance with the results of our study. We found a significant increase in main MMO as well as a reduction in VAS at the twelve-month followup. The increase in these values at the six-month follow-up was statistically non-significant.

\section{MRI}

Kurita et al studied disc morphology through MRI after arthroscopic and nonsurgical treatment and found no significant difference in progression, or improvement in these two groups. Their study also showed that an increase in disc mobility occurred more often after arthroscopic surgery than after nonsurgical treatment at a twenty-month follow-up (12).

Sato et al reported a persistent disc displacement in patients with DDwR of TMJ after treatment of IA HA application. Nevertheless, the clinical symptoms concurrently improved (26). An MRI-based increase in the width of posterior and superior spaces of affected joints after ALL were described in literature.

In the study by Ohnuki et al, comprising four different treatment modalities, only $10 \%$ of the joints became DDwR and the other joints remained DDwR despite the treatment. Upon one-year control MRI, all TMJs had mobile discs. After arthroscopic surgery, the discs were located more anteriorly and also the deformity of the disc advanced when compared with the pre-treatment state. He concluded that the position and deformity of the disc do not need to necessarily improve to get the improvement of signs and symptoms. On the contrary, he proposed that the increase in disc mobility is supposed to be crucial (22). In an eight-year observational study using CT and MRI control by Schiffman et al., from the total of 789 baseline joint-specific soft tissue diagnoses of DD, 598 (76 \%) joints showed no change, 109 (14\%) demonstrated progression, and $82(10 \%)$ had reversal. Schiffman et al. state that currently, we cannot predict which individuals will progress, and at the same time no treatments, including TMJ surgery, can predictably prevent progression of either soft or hard tissue disor- 
ders. Therefore, the current clinical practice should focus on the symptom management rather than on structural intervention for TMJ (27). When treating symptomatic disc displacement of TMJ using ALL, Da Silva et al. found improvement in disc position in 37 cases of TMJ $(68.51 \%)$, the same disc position in 13 cases of TMJ $(24.07 \%)$ and worsened disc position in 4 cases of TMJ (7.42\%) upon six-month follow-up MRI (6).

Our MRI results are consistent with the studies mentioned above. In our study, we found an improvement in the disk position or recapture of the disc's anatomical position in 34 cases (23.44 \%). However, neither ALL or IA HA were able to correct the disc's position in most of the cases. Despite the disc malpositions, there was a significant improvement in clinical symptoms in these patients. No MRI-confirmed improvement was documented in 108 cases $(74.5 \%)$, while the state stayed stationary in 81 patients $(55.9 \%)$, and progressed in 27 cases $(18.6 \%))$. The improvement in clinical symptoms was not primarily related to the improvement in disc's position. In spite of frequent absence of MRI-confirmed improvement, there were only few patients resistant to therapy. This is in support of the presumption that no disc repositioning is needed to improve TMJ function and relieve the patients' symptoms. There is a current trend emerging not necessarily focused on repositioning of the articular disc. Similarly, as in Ohnuki's study, we state that the function of the disc, in terms of its mobility, is more important than its position (22).

Besides, it has been shown clinically that in displaced discs, the posterior band becomes thicker and a cartilage-resembling structure is formed (23). This so-called pseudo-disc formation could enhance the adaptive capacity and bear additional functional loads. According to Bristela, in a study group with pseudodisc, more joints showed normal translation compared to baseline evaluation. Some authors found the presence of pseudo-disc in almost half of the temporomandibular joints with DDwR (4).

We can speculate that chronic overload and overwhelmed compensatory capacity of the joint with a combination of DD could be responsible for the fibrous formation and overbuild of supportive articular structures leading to change in joint stress management.

ALL proved to be superior to HA IA application in both followed clinical parameters. There was no significant difference in MRI results for both groups. Additionally, when the therapy was initiated, the starting MMO and VAS values were less favorable for ALL than for the HA group.

This study had some limitations. First, it was a retrospective study. We were restricted in terms of previously collected data. Therefore, no additional changes to therapy or patient management could possibly be implemented. Secondly, there was no placebo control group. This point is partially justifiable. It would be unethical to treat suffering patients with placebo instead of using proven treatment modalities.

In conclusion, we state, that both ALL and HA IA application are successful and safe methods in the treatment of TMJ ID. A larger and long-term prospective controlled study will be needed to acquire more relevant and detailed information about the intraarticular changes and joint chronic transformation in studied treatment modalities.

\section{References}

1. Abboud WA, Givol N, Yahalom R. Arthroscopic lysis and lavage for internal derangement of the temporomandibular joint. Ann Maxillofac Surg 2015; 5 (2): 158-162: doi.10.4103/2231-0746.175754.

2. Al-Moraissi EA. Open versus arthroscopic surgery for the management of internal derangement of the temporomandibular joint: a meta-analysis of the literature. Internat J Oral Maxillofac Surg 2015; 44 (6): 763-770. doi: 10.1016/j.ijom.2015.01.024.

3. Altman R, Manjoo A, Fierlinger A et al. The mechanism of action for hyaluronic acid treatment in the osteoarthritic knee: a systematic review. BMC Musculoskelet Dis 2015; 16 (1). doi: 10.1186/s12891-015-0775-z.

4. Bristela M, Schmid-Schwap M, Eder J et al. Magnetic resonance imaging of temporomandibular joint with anterior disk dislocation without reposition - long-term results. Clin Oral Investig 2017; 21 (1): 237-245. doi: 10.1007/s00784-016-1800-9.

5. Bronstein SL, Merrill RG. Clinical staging for TMJ internal derangement: application to arthroscopy. J Craniomandib Disord 1992; 6: 7-15.

6. da Silva PA, Moreira LD, Freire FS. The Disc Positioning After Arthroscopic Lysis and Lavage of the Temporomandibular Joint. J Pain Relief 2016; 5: 224. doi: 10.4172/2187-0846.1000224.

7. Edwin YW, Kenneth AF. MRI of temporomandibular joint disorders. Applied Radiology; https://appliedradiology.com/articles/mri-of-temporomandibular-joint-disorders.

8. Gencer ZK, Ozkiris M, Okur A et al. A comparative study on the impact of intra-articular injections of hyaluronic acid, tenoxicam and betametazon on the relief of temporomandibular joint disorder complaints. J Cranio-Maxillofac Surg 2014; 42 (7): 1117-1121. doi: 10.1016/j. jems.2014.01.041.

9. Halmova K, Holly D, Stanko P. The influence of cranio-cervical rehabilitation in patients with myofascial temporomandibular pain disorders. Bratisl Med J 2017; 118 (11): 710-713. doi: 10.4149/BLL_2017_134.

10. Chang CL, Wang DH, Yang MC et al. Functional disorders of the temporomandibular joints: Internal derangement of the temporomandibular joint. Kaohsiung J Med Sci 2018; 34 (4): 223-230. https://doi. org/10.1016/j.kjms.2018.01.004.

11. Korkmaz YT, Altıntas NY, Korkmaz FM et al. Is Hyaluronic Acid Injection Effective for the Treatment of Temporomandibular Joint Disc Displacement With Reduction? J Oral Maxillofac Surg 2016; 74 (9): 1728 1740. doi: 10.1016/j.joms.2016.03.005.

12. Kurita H, Chen Z, Uehara S et al. Comparison of Imaging FollowUp Between Joints With Arthroscopic Surgery (Lysis and Lavage) and Those With Nonsurgical Treatment. J Oral Maxillofac Surg 2007; 65 (7): 1309-1314. doi: 10.1016/j.joms.2006.10.040.

13. Larheim TA. Role of Magnetic Resonance Imaging in the Clinical Diagnosis of the Temporomandibular Joint. Cells Tissues Organs 2005; 180 (1): 6-21. doi: 10.1159/000086194.

14. Long X, Chen G, Cheng AH et al. A Randomized Controlled Trial of Superior and Inferior Temporomandibular Joint Space Injection with Hyaluronic Acid in Treatment of Anterior Disc Displacement Without Reduction. J Oral Maxillofac Surg 2009; 67 (2): 357-361. doi: 10.1016/j. joms.2008.09.014.

15. Long X, Chen G, Cheng AH et al. Superior and inferior temporomandibular joint space injection with hyaluronic acid in treatment of anterior disk displacement without reduction. J Oral Maxillofac Surg 2009; 67: $357-361$. 
16. Machon V, Lukasova V. Př́íspěvek k etiologii onemocnění temporomandibulárního kloubu. Prakt Zub Lek 2005; 53 (6): 111-114.

17. Malachovsky I, Statelova D, Sitar J. Hyaluronic acid in the treatment of disturbances of temporomandibular joint. Stomatológ 2001; 11 (4): 28-29.

18. Manfredini D, Piccotti F, Guarda-Nardini L. Hyaluronic Acid In the Treatment of TMJ Disorders: A Systematic Review of the Literature. CRANIO® 2010; 28 (3): 166-176. doi:10.1179/crn.2010.023.

19. Mracna J. Rozdelenie, klinické prejavy a diagnostika ochorení temporomandibulárneho kíbu. Bratisl Med J 1992; 93 (10): 550.

20. Murakami K. Rationale of arthroscopic surgery of the temporomandibular joint. J Oral Biol Craniofac Res 2013; 3 (3): 126-134. doi: 10.1016/j.jobcr.2013.07.002

21. Murakami K. I, Tsuboi Y, Bessho K. et al. Outcome of arthroscopic surgery to the temporomandibular joint correlates with stage of internal derangement: five-year follow-up study. Brit J Oral Maxillofac Surg 1998; 36 (1): 30-34. doi: 10.1016/s0266-4356(98)90744-6.

22. Ohnuki T, Fukuda M, Nakata A et al. Evaluation of the position, mobility, and morphology of the disc by MRI before and after four different treatments for temporomandibular joint disorders. Dentomaxillofac Radiol 2006; 35 (2): 103-109. doi: 10.1259/dmfr/25020275.

23. Perez del Palomar A, Doblaré M. Influence of unilateral disc displacement on the stress response of the temporomandibular joint discs during opening and mastication. J Anat 2007; 211 (4): 453-463. doi: 10.1111/j.1469-7580.2007.00796.x.
24. Sato S, Nasu F, Motegi K. Analysis of kinesiograph recordings and masticatory efficiency after treatment of non-reducing disk displacement of the temporomandibular joint. J Oral Rehabil 2003; 30: 708-713.

25. Sato S, Oguri S, Yamaguchi K et al. Pumping injection of hyaluronic acid for patients with nonreducing disc displacement of the temporomandibular joint: two year follow-up. J Craniomaxillofac Surg 2001; 29: 89-93.

26. Sato S, Sakamoto M, Kawamura $\mathbf{H}$ et al. Disc position and morphology in patients with nonreducing disc displacement treated by injection of sodium hyaluronate. Int J Oral Maxillofac Surg 1999; 28: 253-257.

27. Schiffman EL, Ahmad M, Hollender L et al. Longitudinal Stability of Common TMJ Structural Disorders. J Dent Res 2016; 96 (3): 270-276. doi: 10.1177/0022034516679396.

28. Schiffman E, Ohrbach R, Truelove E et al. Diagnostic Criteria for Temporomandibular Disorders (DC/TMD) for Clinical and Research Applications: recommendations of the International RDC/TMD Consortium Network and Orofacial Pain Special Interest Group. J Oral Facial Pain Headache 2014; 28 (1): 6-27. doi: 10.11607/jop.1151.

29. Tvrdy P, Heinz P, Pink R. Arthrocentesis of the temporomandibular joint: A review. Biomed Pap Med Fac Univ Palacky Olomouc 2015; 159 : 31-34. doi: 10.5507/bp.2013.026.

30. White RD. Arthroscopic lysis and lavage as the preferred treatment for internal derangement of the temporomandibular joint. J Oral Maxillofac Surg 2001; 59: 313. doi: 10.1053/joms.2001.21002.

Received December 12, 2019. Accepted February 17, 2020. 